\title{
Consumo, digestibilidade e balanço de nitrogênio em ovinos alimentados com rações contendo torta de Crambe
}

[Intake, digestibility and nitrogen balance in sheep fed with diets containing Crambe cake]

\author{
I.Y. Mizubuti ${ }^{1}$, M.A. Syperreck ${ }^{1 *}$, E.L.A. Ribeiro ${ }^{1}$, E.S. Pereira ${ }^{2}$, A.P. Pinto ${ }^{2}$, O.P.P. Prado ${ }^{1}$, \\ E.L.T. Peixoto ${ }^{3}$, A.R.P. Parra ${ }^{1}$, F.L. Massaro Júnior ${ }^{1}$, G.L. Guerra ${ }^{4}$ \\ ${ }^{1}$ Universidade Estadual de Londrina - Londrina, PR \\ ${ }^{2}$ Universidade Federal do Ceará - Fortaleza, CE \\ ${ }^{3}$ Universidade Federal do Sul e Sudeste do Pará - Nova Marabá, Marabá, PA \\ ${ }^{4}$ Aluna de pós-graduação - Universidade Estadual de Londrina - Londrina, PR
}

\begin{abstract}
RESUMO
O objetivo deste trabalho foi avaliar os efeitos da utilização de torta de crambe na ração de ovinos sobre o consumo e a digestibilidade. $\mathrm{O}$ delineamento experimental utilizado foi o quadrado latino $4 \times 4$ com quatro animais, quatro períodos e quatro tratamentos. Os tratamentos avaliados foram rações com $30 \%$ de silagem de sorgo e $70 \%$ de torta de crambe (30:70) e rações com $70 \%$ de silagem de sorgo e $30 \%$ de torta de crambe (70:30) em cordeiros sob consumo voluntário e controlado. Os animais que receberam a ração com a relação 30:70 consumiram mais matéria seca (MS), proteína bruta (PB), extrato etéreo (EE) por $\mathrm{kg} / \mathrm{dia}$, \% PV/dia e g/kg PV ${ }^{0,75}$. A relação 30:70 proporcionou maior coeficiente de digestibilidade de MS e FDN. Os animais no regime voluntário apresentaram maior consumo de MS, PB, EE por $\mathrm{kg} / \mathrm{dia}, \%$ $\mathrm{PV} /$ dia e $\mathrm{g} / \mathrm{kg} \mathrm{PV}^{0,75}$. A torta de crambe apresentou coeficientes de digestibilidade superiores à silagem de sorgo, independentemente do regime de consumo. A relação 30:70 apresentou diferença para o $\mathrm{N}$-ingerido e N-absorvido. A torta de crambe apresenta alta digestibilidade dos nutrientes e aceitação pelos animais quando associada à silagem de sorgo.
\end{abstract}

Palavras-chave: Crambe abyssinica, agroindústria, alimento alternativo, valor nutritivo

\begin{abstract}
The aim of this study was to evaluate the effects of using crambe cake in diets for sheep on intake and digestibility. The experimental design was a $4 \times 4$ Latin square with four animals, four periods and four treatments. The treatments were diets with $30 \%$ sorghum silage and $70 \%$ crambe cake (30:70) and a diet with 70\% sorghum silage and 30\% crambe cake (70:30) in lambs under the voluntary and controlled intake. The animals who fed a diet with a 30:70 ratio consumed more dry matter (DM), crude protein $(C P)$, ether extract (EE) per $\mathrm{kg} /$ day, \%BW/day and $\mathrm{g} / \mathrm{kgP} \mathrm{V}^{0,75}$. The ration provided higher digestibility of $D M$ and NDF. The animals in the voluntary regime presented higher consumption of DM, CP, EE per $\mathrm{kg} /$ day, \%BW/day and $\mathrm{g} / \mathrm{kgPV}^{0,75}$. The cake crambe presented digestibility coefficient higher than sorghum silage, regardless of the regime intake. The 30:70 ration presented a difference in $N$ intake and $N$ absorbed. The cake crambe has high nutrient digestibility and acceptance by animals when associated with sorghum silage.
\end{abstract}

Keywords: Crambe abyssinica, Agribusiness, alternative food, nutritional value

Recebido em 27 de julho de 2015

Aceito em 15 de novembro de 2015

*Autor para correspondência (corresponding author)

E-mail: syperreck@gmail.com 


\section{INTRODUÇÃO}

Segundo a Agência Nacional de Petróleo, Gás Natural e Biocombustíveis, o Brasil se encontra entre os maiores produtores e consumidores de biodiesel do mundo, com uma produção nacional de 209 milhões de $\mathrm{m}^{3}$ em 2014 (ANP, 2014).

Com a extração do óleo, são obtidos diversos coprodutos e subprodutos com características que permitem seu uso na nutrição de ruminantes. A composição desses coprodutos e subprodutos varia segundo o cultivar da oleaginosa e o tipo de extração (Mendonça et al., 2015).

O crambe possui elevada concentração de óleo (35 a 37\%) quando comparado com outras oleaginosas e tem sido utilizado para produção de biodiesel na região Centro-Oeste do Brasil (Souza et al., 2009), tornando-se uma cultura inovadora no setor de agronegócio brasileiro.

A diversificação de alimentos provenientes de culturas alternativas na nutrição e na produção de cordeiros, a fim de reduzir custos com a alimentação, tem sido bastante utilizada. A torta de crambe é um coproduto da extração do óleo de crambe por prensagem e apresenta características físicas e químicas que a tornam um alimento com grande potencial de utilização na alimentação de cordeiros, podendo substituir ingredientes proteicos convencionais usados em rações de ruminantes. A utilização desse coproduto propicia menor custo de produção e o reaproveitamento de matéria orgânica de origem vegetal (Mizubuti et al., 2011; Brás, 2011).

O conhecimento da composição química, do consumo e dos valores de digestibilidade tem contribuído significativamente para o desenvolvimento de sistemas de predição do valor nutritivo de alimentos, sendo, por isso, parâmetro importante na avaliação de alimento, principalmente, quando se trata de coprodutos (Van Soest, 1994).

Portanto, este trabalho foi realizado com o objetivo de avaliar o consumo, a digestibilidade dos nutrientes e o balanço de nitrogênio em ovinos alimentados com rações contendo silagem de sorgo e torta de crambe, em diferentes relações de volumoso:concentrado, 30:70 e 70:30, em cordeiros sob regimes de consumo voluntário e controlado.

\section{MATERIAL E MÉTODOS}

O experimento foi conduzido no Laboratório de Metabolismo Animal do Departamento de Zootecnia, da Universidade Estadual de Londrina (UEL), Paraná, após aprovação prévia do projeto pelo Comitê de Ética da UEL, sob número de protocolo CEEA 60/2010.

Foram utilizados quatro ovinos machos, castrados, com peso médio de $26,0 \mathrm{~kg}$, e vermifugados com produto à base de closantel, na dosagem de $1 \mathrm{~mL}$ para cada $10 \mathrm{~kg}$ de peso vivo, conforme recomendação do fabricante. Os animais experimentais permaneceram em piquetes recebendo silagem de sorgo durante 12 dias e, em seguida, foram identificados e instalados aleatoriamente em gaiolas metabólicas individuais, providas de comedouro, bebedouro e cocho próprio para suplemento mineral.

O delineamento experimental utilizado foi o quadrado latino $4 \times 4$, com quatro animais e quatro períodos. Os alimentos avaliados foram a silagem de sorgo (SS) e a torta de crambe (TC) em dietas em proporções de 30:70 e 70:30, respectivamente (Tab. 1), fornecidos aos animais em regime de consumo voluntário $(\mathrm{CV})$ e consumo controlado (CC).

O experimento teve duração de 48 dias, divididos em quatro períodos experimentais de 12 dias, sendo sete dias destinados à adaptação às dietas experimentais e cinco dias de coletas de amostras para determinação da digestibilidade. No período de coleta, foi mensurado o consumo de alimentos. Os animais foram submetidos à pesagem individual no início e no fim de cada período experimental.

As dietas foram fornecidas duas vezes ao dia, às $7 \mathrm{~h} 30 \mathrm{e}$ às $16 \mathrm{~h}$, e as sobras foram recolhidas e pesadas diariamente, antes da alimentação matinal, para determinação do consumo. No regime de consumo voluntário, o fornecimento de alimentos foi ajustado diariamente por diferença entre o alimento fornecido e as sobras, por animal, de modo a manter sobras de $10 \%$ do total de matéria seca fornecida, garantindo, assim, o consumo voluntário. No regime de consumo controlado, foi calculada a média de consumo de todos os animais, durante o período de adaptação, e a ração fornecida foi de $90 \%$ dessa média. Água e sal mineralizado estiveram 
à disposição dos animais em cochos separados. $\mathrm{O}$ sal mineralizado continha os seguintes níveis de garantia/kg: $\mathrm{Ca}, 150 \mathrm{~g} ; \mathrm{P}, 65 \mathrm{~g} ; \mathrm{Na}, 107 \mathrm{~g} ; \mathrm{Mg}$,
$6.000 \mathrm{mg} ; \mathrm{S}, 12 \mathrm{~g} ; \mathrm{Co}, 175 \mathrm{mg} ; \mathrm{Cu}, 100 \mathrm{mg}$; I,175mg; Mn, 1.400mg; Se, 27mg; Zn, 6.000mg; Fe, 1.000mg; F, 650mg.

Tabela 1. Composição bromatológica dos ingredientes e das dietas experimentais utilizadas na alimentação de ovinos

\begin{tabular}{lcccc}
\hline & \multicolumn{2}{c}{ Ingredientes } & \multicolumn{2}{c}{ Dietas } \\
\cline { 2 - 5 } Componentes (\%) & SS & TC & $70: 30^{1}$ & $30: 70^{2}$ \\
\hline Matéria seca & 31,20 & 93,10 & 49,79 & 74,57 \\
Proteína bruta & 6,80 & 28,30 & 13,30 & 21,09 \\
Extrato etéreo & 1,80 & 24,30 & 8,60 & 17,60 \\
Fibra em detergente neutro & 64,20 & 68,00 & 66,90 & 65,40 \\
Fibra em detergente ácido & 45,20 & 44,50 & 42,20 & 40,00 \\
Carboidratos totais & 85,90 & 42,60 & 72,93 & 55,50 \\
Matéria orgânica & 94,60 & 95,20 & 94,80 & 95,10 \\
Matéria mineral & 5,40 & 4,80 & 5,20 & 4,90 \\
\hline
\end{tabular}

$\mathrm{SS}=$ silagem de sorgo; $\mathrm{TC}=$ torta de crambe.

${ }^{1}$ Dieta com $70 \%$ de SS e $30 \%$ de TC; ${ }^{2}$ dieta com $30 \%$ de SS e $70 \%$ de TC.

Durante o período de coleta, foram retiradas amostras das rações fornecidas e das sobras para elaboração de amostras compostas por animal, as quais foram acondicionadas em sacos plásticos devidamente identificados e guardadas em freezer para posteriores análises.

A coleta de fezes foi realizada duas vezes ao dia, às $7 \mathrm{~h} 30$ e às $16 \mathrm{~h} 30$, por meio de bolsas coletoras, acopladas aos animais. As fezes de cada animal foram pesadas diariamente, e, após homogeneização do material, foi retirada uma alíquota diária, em torno de $20 \%$ do total, para preparo de uma amostra composta por animal. As amostras de fezes foram colocadas em sacos plásticos, identificadas e armazenadas em freezer, para análises posteriores. Essas amostras, juntamente com as amostras dos alimentos fornecidos e das sobras, foram submetidas à présecagem a $55^{\circ} \mathrm{C}$, por 72 horas. Em seguida, foram processadas em moinho tipo "Willey", com peneira de $1 \mathrm{~mm}$, e acondicionadas em recipientes de plástico com tampa de polietileno.

As análises de matéria seca (MS), material mineral $(\mathrm{MM})$, proteína bruta $(\mathrm{PB})$, extrato etéreo (EE), fibra em detergente neutro (FDN) e fibra em detergente ácido (FDA) dos ingredientes e das dietas experimentais (Tab. 1) foram efetuadas conforme metodologias descritas por Mizubuti et al. (2009). Os teores de carboidratos totais (CT) foram calculados segundo recomendações de Sniffen et al. (1992): $\mathrm{CT}=100-(\% \mathrm{~PB}+\% \mathrm{EE}+\%$ Cinzas $) . \mathrm{A}$ determinação do consumo e do coeficiente de digestibilidade dos nutrientes das dietas foi obtida conforme descritas por Coelho da Silva e Leão (1979).

Os dados obtidos foram submetidos à análise de variância, e as médias comparadas pelo teste de Tukey a $5 \%$ de probabilidade, utilizando-se o programa SAS. Adicionalmente, foram feitos estudos de contraste visando testar as diferenças entre os regimes alimentares (voluntário vs. restrito) e entre as relações de silagem de sorgo:torta de crambe na dieta (70:30 e 30:70).

\section{RESULTADOS E DISCUSSÃO}

Dieta com a relação silagem de sorgo (SS): torta de crambe (TC) de 30:70 influenciou $(\mathrm{P}>0,05)$ o consumo de matéria seca (MS), proteína bruta (PB) e extrato etéreo (EE) ( $\mathrm{g} / \mathrm{dia}, \% \mathrm{PV}$ e g/kg $\mathrm{PV}^{0,75}$ ), proporcionando maior consumo destes nutrientes em relação aos animais alimentados com dieta contendo a relação 70:30 (Tab. 2).

Os regimes alimentares também influenciaram $(\mathrm{P}<0,05)$ o consumo de nutrientes, e animais sob o regime de consumo voluntário apresentaram maior consumo de MS, PB (g/dia, \% PV e g/kg $\mathrm{PV}^{0,75}$ ) e EE (g/dia e g/kg PV ${ }^{0,75}$ ) (Tab. 2). 
Tabela 2. Consumo de nutrientes em ovinos considerando-se a relação de silagem de sorgo e torta de crambe (70:30 e 30:70) e os regimes alimentares (voluntário e controlado)

\begin{tabular}{|c|c|c|c|c|c|c|c|}
\hline \multicolumn{8}{|c|}{ Consumo de nutrientes $(\mathrm{kg} / \mathrm{dia})$} \\
\hline Tratamento & MS & PB & $\mathrm{EE}$ & FDN & FDA & CT & $\mathrm{MO}$ \\
\hline Relação 70:30 & $0,67 \mathrm{~b}$ & $0,13 b$ & $0,08 \mathrm{~b}$ & 0,70 & 0,31 & 0,61 & 0,71 \\
\hline Relação 30:70 & $1,01 \mathrm{a}$ & $0,22 \mathrm{a}$ & $0,16 \mathrm{a}$ & 0,84 & 0,19 & 0,51 & 0,91 \\
\hline Voluntário & $0,94 \mathrm{a}$ & $0,19 \mathrm{a}$ & $0,14 \mathrm{a}$ & 0,83 & 0,22 & 0,62 & 0,95 \\
\hline Controlado & $0,74 b$ & $0,15 b$ & $0,10 \mathrm{~b}$ & 0,71 & 0,27 & 0,49 & 0,76 \\
\hline $\mathrm{CV}^{1}{ }^{1}$ & 9,8 & 11,2 & 10,9 & 10,6 & 11,2 & 10,8 & 10,9 \\
\hline \multicolumn{8}{|c|}{ Consumo de nutriente $(\% \mathrm{PV} /$ dia $)$} \\
\hline Tratamento & MS & PB & $\mathrm{EE}$ & FDN & FDA & CT & MO \\
\hline Relação 70:30 & $2,53 \mathrm{~b}$ & $0,48 b$ & $0,30 \mathrm{~b}$ & 2,63 & 1,16 & 2,31 & 2,45 \\
\hline Relação 30:70 & $3,86 a$ & $0,84 a$ & $0,63 \mathrm{a}$ & 3,23 & 0,73 & 1,93 & 3,49 \\
\hline Voluntário & $3,58 \mathrm{a}$ & $0,74 a$ & 0,54 & 3,17 & 0,85 & 2,37 & 3,50 \\
\hline Controlado & $2,81 b$ & $0,57 b$ & 0,39 & 2,68 & 1,03 & 1,87 & 2,79 \\
\hline $\mathrm{CV} \%$ & 8,8 & 10,2 & 11,2 & 12,2 & 11,9 & 11,4 & 11,0 \\
\hline \multicolumn{8}{|c|}{ Consumo de nutriente $\left(\mathrm{g} / \mathrm{kg} \mathrm{PV}^{0,75}\right)$} \\
\hline Tratamento & MS & PB & $\mathrm{EE}$ & FDN & FDA & $\mathrm{CT}$ & $\mathrm{MO}$ \\
\hline Relação 70:30 & $57,6 \mathrm{~b}$ & $10,9 b$ & $6,8 \mathrm{~b}$ & 59,8 & 26,4 & 52,5 & 53,6 \\
\hline Relação 30:70 & $87,5 \mathrm{a}$ & $19,0 \mathrm{a}$ & $14,3 \mathrm{a}$ & 73,1 & 16,4 & 43,7 & 79,78 \\
\hline Voluntário & $81,2 \mathrm{a}$ & $16,9 a$ & $12,3 \mathrm{a}$ & 72,0 & 19,3 & 53,8 & 80,6 \\
\hline Controlado & $63,9 b$ & $13,0 \mathrm{~b}$ & $8,88 \mathrm{~b}$ & 60,9 & 23,5 & 42,4 & 50,9 \\
\hline $\mathrm{CV} \%{ }^{1}$ & 8,9 & 11,7 & 11,7 & 10,9 & 11,5 & 10,6 & 10,5 \\
\hline
\end{tabular}

${ }^{\mathrm{C}} \mathrm{CV}$ : coeficiente de varação. Médias seguidas de letras diferentes na coluna diferem entre si $(\mathrm{P}<0,05)$ pelo teste Tukey.

O maior consumo de MS apresentado pelos animais alimentados com a dieta contendo relação 30:70 pode estar relacionado com a digestibilidade da MS da ração. Segundo Faria e Mattos (1995), a máxima ingestão de MS ocorre quando a digestibilidade da dieta se encontra entre 66 e $68 \%$. Considerando-se a quantidade de torta de crambe na dieta, outros fatores, como a ação direta dos hormônios intestinais, a oxidação de ácidos graxos no fígado e a aceitabilidade das fontes utilizadas (Allen, 2000), também podem ter influenciado o consumo de MS.

A quantidade de torta de crambe (70\%) presente na dieta pode ter influenciado o consumo devido às características dos ácidos graxos presentes nesse alimento, que são liberados lentamente durante a fermentação ruminal (Palmquist e Mattos, 2006). Além disso, a torta de crambe pode ter fornecido ambiente propício às atividades dos microrganismos ruminais por apresentar ácidos graxos de cadeia curta e $\mathrm{pH}$ adequados (Brás, 2011), favorecendo a digestibilidade e o consumo de MS. Resultados semelhantes foram encontrados por Brás (2011), que relatou CMS de $0,92 \mathrm{~kg} / \mathrm{dia}$ em animais recebendo rações com torta de crambe.

O menor consumo de MS apresentado para a relação 70:30 pode ser explicado por um fluxo reduzido da digesta através do trato gastrintestinal, pois, segundo Mertens (1994), quando os animais se alimentam de dietas palatáveis, porém altas em volume e baixas em concentração energética, o consumo é limitado por alguma restrição na capacidade do trato digestivo. Dessa maneira, o consumo pode ter sido limitado pelo tempo despendido para processar ou mastigar a silagem em partículas menores e também pela estrutura da fibra.

Outro fator que pode ter influenciado o consumo nesta relação é a característica da fibra da silagem de sorgo, bem como o percentual de extrato etéreo da ração, que pode influenciar o tempo médio de retenção da digesta (Pereira et al., 2009) e, como consequência, reduzir o consumo de matéria seca.

Os valores de consumo de proteína bruta em $\mathrm{kg} /$ dia são maiores que os recomendados pelo AFRC (1998), o que confirma a boa aceitabilidade da torta de crambe pelos cordeiros. Entretanto, como as relações avaliadas não prejudicaram o consumo de proteína, isso pode indicar que a torta de crambe é um alimento com grande potencial para a nutrição de animais ruminantes, pois, em ambos os tratamentos, não prejudicou o consumo dos nutrientes e foram atendidas as exigências dos animais. 
$\mathrm{O}$ maior consumo de EE na dieta com relação 30:70 pode ser devido ao fato de que as sementes de oleaginosas como a torta de crambe podem fornecer energia e elevados teores de proteína bruta, podendo ser consideradas como fonte de lipídeos parcialmente protegida, visto que as gotículas de lipídeos nessas sementes encontramse inseridas na matriz proteica, conferindo-lhes proteção natural (Urano, 2009). Essa característica também proporciona, segundo Valadares Filho e Pina (2006), redução da metanogênese, da concentração de amônia ruminal e do aumento da produção de propionato.

O consumo de FDN em \% PV nos animais recebendo as dietas com as relações 70:30 e $30: 70$ foi superior a $1,2 \%$ que é o recomendado. Apesar do elevado consumo de FDN em \% PV dos animais alimentados com as relações 70:30 e 30:70, este não foi um fator limitante no controle da ingestão das rações. De acordo com Van Soest (1994), forragens com altos níveis de fibra permanecem por mais tempo no trato gastrintestinal, gerando menor consumo voluntário, fato que pode ter causado o menor consumo da ração contendo $70 \%$ de silagem de sorgo.

Com relação ao regime alimentar, o maior consumo de matéria seca, proteína bruta e extrato etéreo dos animais sob regime de consumo voluntário pode ser em razão da capacidade seletiva mantida por esses animais de separar os alimentos e, portanto, consumir mais, quando comparados aos animais em regime de consumo controlado; dessa forma, fica evidente o efeito da restrição alimentar para o consumo dos nutrientes das rações experimentais (kg/dia, \% $\mathrm{PV} /$ dia e $\mathrm{g} / \mathrm{kg} \mathrm{PV}^{0,75}$ ). Outro fator depende da regulação do consumo, o qual é complexo e inclui limitações físicas, controle fisiológico e fatores psicogênicos (Doughterty e Collins, 2003).

Houve efeito $(\mathrm{P}<0,05)$ das relações 30:70 e 70:30 sobre a digestibilidade da MS e da FDN, e a relação 30:70 apresentou os maiores coeficientes (Tab. 3).

Tabela 3. Coeficiente de digestibilidade dos nutrientes das rações utilizadas na alimentação de ovinos contendo diferentes relações de silagem de sorgo e torta de crambe (70:30 e 30:70) e os regimes alimentares (voluntário vs. controlado)

\begin{tabular}{lccccccc}
\hline \multicolumn{7}{c}{ Coeficiente de digestibilidade (\%) } \\
\hline Tratamento & MS & PB & EE & FDN & FDA & CT & MO \\
\hline Relação 70:30 & $50.06 \mathrm{~b}$ & 70.72 & 87.63 & $65.82 \mathrm{~b}$ & 53.95 & 66.03 & 68.55 \\
Relação 30:70 & $67.34 \mathrm{a}$ & 66.72 & 89.86 & $72.44 \mathrm{a}$ & 52.50 & 62.93 & 69.44 \\
\hline Voluntário & $63.50 \mathrm{a}$ & 69.53 & 86.00 & 67.56 & $47.69 \mathrm{~b}$ & 62.61 & $65.97 \mathrm{~b}$ \\
Controlado & $53.90 \mathrm{~b}$ & 67.91 & 91.48 & 70.70 & $58.76 \mathrm{a}$ & 66.35 & $72.02 \mathrm{a}$ \\
\hline CV\% & 6,18 & 5,61 & 3,6 & 3,6 & 8,8 & 8,0 & 5,37
\end{tabular}

MS: matéria seca; PB: proteína bruta; EE: extrato etéreo; FDN: fibra em detergente neutro; FDA: fibra em detergente ácido; MO: matéria orgânica; CT: carboidratos totais. Médias seguidas de letras diferentes na coluna, dentro de relações volumoso:concentrado ou dentro de regimes alimentares, diferem entre si $(\mathrm{P}<0,05)$ pelo teste Tukey.

Os maiores coeficientes de digestibilidade da MS e da FDN na dieta com relação 30:70 podem ser devido à digestibilidade do alimento volumoso, o qual depende da atividade dos microrganismos sobre a digestão da celulose no rúmen. Se a esse volumoso for adicionado um concentrado que contém nutrientes benéficos aos microrganismos, isso resulta na melhoria da digestibilidade aparente da celulose do volumoso (Gonçalves et al., 2009). Os resultados mostraram que a torta de crambe promoveu efeito benéfico à microflora ruminal, pois é um alimento rico em proteína e extrato etéreo, e interferiu positivamente na digestibilidade dos nutrientes da silagem de sorgo.

O maior coeficiente de digestibilidade do EE e da FDN na silagem de sorgo para o consumo controlado pode ser em razão da utilização de alimentos ricos em lipídeos, uma vez que estes aumentam a densidade energética da dieta sem aumentar os riscos de ocorrências de acidose ruminal (Fernandes et al., 2002). A adição desses nutrientes à dieta em quantidades acima de $7 \%$ do total da matéria seca pode prejudicar a digestibilidade das fibras (hemicelulose e celulose) e demais nutrientes no rúmen e/ou 
provocar distúrbios metabólicos, comprometendo o desempenho animal.

Hess et al. (2008), entretanto, constataram que $9,4 \%$ de adição de lipídeos na matéria seca não afetaram a digestibilidade de outros componentes da dieta, o que foi observado com a relação $30: 70$, que continha $17,6 \%$ de extrato etéreo.

Para os regimes alimentares, o consumo voluntário apresentou coeficiente de MS superior, e os coeficientes de FDA e MO foram superiores para o consumo controlado. A principal causa da variação na digestibilidade da dieta para o regime alimentar controlado é o tempo de retenção de partículas no rúmen. Vários fatores podem contribuir para a diversidade nas respostas, desde o nível de concentrado da dieta até mecanismos de adaptação na absorção de nutrientes. É possível observar diferentes respostas de digestibilidade em animais mantidos sob restrição alimentar, não sendo elucidados todos os fatores que levam a essas variações, especialmente com relação à diminuição na digestibilidade (Doreau et al., 2003).

A torta de crambe apresentou coeficientes de digestibilidade aparente superiores à silagem de sorgo, independentemente do regime alimentar (Tab. 4). Entretanto, no regime de consumo, o coeficiente de MS foi superior para o regime controlado.

A silagem de sorgo apresentou coeficientes de digestibilidade superiores para MS no consumo voluntário, e no consumo controlado para EE e FDN. Embora os coeficientes de digestibilidade da silagem tenham sido menores que os da torta de crambe, eles são considerados altos e satisfatórios.

Tabela 4. Coeficiente de digestibilidade aparente (CDA) dos nutrientes em ovinos alimentados com silagem de sorgo e torta de crambe

\begin{tabular}{ccccccccc}
\hline \multirow{2}{*}{ Ingrediente } & \multirow{2}{*}{ Regime } & \multicolumn{7}{c}{ Coeficiente de digestibilidade aparente (\%) } \\
\cline { 3 - 8 } & & MS & PB & EE & FDN & FDA & MO & CT \\
\hline Silagem & Controlado & $48,9 \mathrm{~b}$ & 55,4 & $79,7 \mathrm{a}$ & $54,6 \mathrm{a}$ & 48,0 & 54,0 & 57,7 \\
de sorgo & Voluntário & $53,0 \mathrm{a}$ & 55,2 & $75,9 \mathrm{~b}$ & $53,1 \mathrm{~b}$ & 44,6 & 54,5 & 58,1 \\
\hline Torta de & Controlado & $76,1 \mathrm{a}$ & 71,0 & 81,1 & 67,9 & 51,1 & 46,5 & 64,5 \\
crambe & Voluntário & $69,6 \mathrm{~b}$ & 69,6 & 77,0 & 68,1 & 43,8 & 46,3 & 62,2 \\
\hline CV\% & & 3,04 & 9,5 & 2,6 & 3,2 & 8,8 & 8,5 & 4,3 \\
\hline
\end{tabular}

MS: matéria seca (MS); PB: proteína bruta; EE: extrato etéreo; FDN: fibra em detergente neutro; FDA: fibra em detergente ácido; MO: matéria orgânica e CT: carboidratos totais. Médias seguidas de letras diferentes na coluna, dentro de relações volumoso:concentrado ou dentro de regimes alimentares, diferem entre si $(\mathrm{P}<0,05)$ pelo teste Tukey.

O fato de a digestibilidade dos nutrientes da torta de crambe não diferir entre os regimes alimentares pode ser em virtude de os triglicerídeos contidos serem de liberação lenta, o que permitiu bio-hidrogenação mais extensa pelos microrganismos. Além disso, pode ter ocorrido escape de AGI do rúmen, já que estes estariam protegidos no interior das células desse alimento. A liberação lenta de lipídeos presentes em sementes de oleaginosas resulta em efeitos mínimos sobre a digestibilidade da fibra pelos microrganismos (Doreau e Ferlay, 1995). Silva e Nörnberg (2003) destacaram que um bom alimento, com elevada quantidade de lipídeos é aquele que não prejudica o metabolismo ruminal e que, ao mesmo tempo, apresenta elevada digestibilidade.
Considerando-se os resultados de digestibilidade das rações, é importante ressaltar que o suco pancreático de ovinos tem uma forte ação lipolítica, portanto os lipídeos que não são hidrolisados no rúmen ou aqueles que chegam intactos ao intestino dificilmente escapam da digestão, justificando o alto percentual de digestibilidade dos nutrientes da torta de crambe.

A dieta com relação 30:70 apresentou diferença para o N-ingerido e o N-absorvido (Tab. 5), o que corresponde aproximadamente a 196,75 e $150,87 \mathrm{~g}$ de $\mathrm{PB} / \mathrm{animal} / \mathrm{dia}$, respectivamente. Observou-se que todos os tratamentos apresentaram balanço de nitrogênio (BN) positivo, indicando que os animais estavam sob condições alimentares de ganho de $\mathrm{N}$. 
Tabela 5. Balanço de nitrogênio (g/dia) nos ovinos considerando-se a relação de silagem de sorgo e torta de crambe (70:30 e 30:70) e os regimes alimentares (voluntário vs. controlado)

\begin{tabular}{lcccc} 
Tratamento & $\begin{array}{c}\text { Nitrogênio } \\
\text { ingerido }\end{array}$ & $\begin{array}{c}\text { Nitrogênio } \\
\text { eliminado }\end{array}$ & $\begin{array}{c}\text { Nitrogênio } \\
\text { absorvido }\end{array}$ & $\begin{array}{c}\text { Balanço de } \\
\text { nitrogênio }\end{array}$ \\
\hline Relação 70:30 & $20,6 \mathrm{~b}$ & 2,6 & $18,81 \mathrm{~b}$ & 13,61 \\
Relação 30:70 & $35,0 \mathrm{a}$ & 5,0 & $31,39 \mathrm{a}$ & 22,20 \\
CV\% & 5,5 & 3,2 & 7,1 & 5,0 \\
\hline Voluntário & 31,48 & 4,3 & $28,66 \mathrm{a}$ & 18,93 \\
Controlado & 24,1 & 3,2 & $16,88 \mathrm{~b}$ & 21,64 \\
\hline
\end{tabular}

Médias seguidas de letras diferentes na coluna, dentro de relações volumoso:concentrado ou dentro de regimes alimentares, diferem entre si $(\mathrm{P}<0,05)$ pelo teste Tukey.

A diferença observada para $\mathrm{N}$-ingerido e $\mathrm{N}$ absorvido da relação 30:70 já era esperada, em virtude da maior quantidade de proteína. $\mathrm{O}$ balanço de nitrogênio (BN) positivo dos tratamentos é um indicativo de que os animais estavam sob condições alimentares de ganho de $\mathrm{N}$. Independentemente dos tratamentos utilizados, não se observaram valores negativos para o $\mathrm{BN}$, indicando que houve retenção de proteína no organismo animal e que o consumo de proteína atendeu satisfatoriamente as exigências proteicas dos animais.

\section{CONCLUSÕES}

A torta de crambe apresenta boa digestibilidade de seus nutrientes e, quando associada à silagem de sorgo, melhora a digestibilidade dos nutrientes desse volumoso. A utilização da torta de crambe numa relação volumoso:concentrado de 30:70 melhora o consumo de nutrientes (kg/dia, \% PV/dia e g/kg PV ${ }^{0,75}$ ) de cordeiros.

\section{AGRADECIMENTO}

Ao engenheiro agrônomo Carlos Pitol e à Fundação MS para Pesquisa e Difusão de Tecnologias Agropecuárias, pela doação da matéria-prima para este estudo.

\section{REFERÊNCIAS}

ALLEN, M.S. Effects of diet on short-term regulation of feed intake by lactating dairy cattle. J. Dairy Sci, v.83, p.1598-1624, 2000.

ANUÁRIO ESTATÍSTICO BRASILEIRO DO PETRÓLEO, GÁS NATURAL E BIOCOMBUSTÍVEIS: 2014. Rio de Janeiro: ANP, 2014. 246p.
BRÁS, P. Caracterização nutricional de coprodutos da extração de óleo em grãos vegetais em dietas de ovinos. 2011. 75f. Dissertação (Mestrado em Produção Animal Sustentável) Programa de Pós-Graduação em Produção Animal Sustentável, Instituto de Zootecnia, Nova Odessa, SP.

DOREAU, M.; FERLAY, A. Effect of dietary lipids on nitrogen metabolism in the rumen: a review. Livest Prod. Sci., v.43, p.97-110, 1995.

DOREAU, M.; MICHALET-DOREAU, B.; GRIMAUD, P.; ATTI, N.; NOZIÈRE, P. Consequences of underfeeding on digestion and absorption in sheep. Small Ruminant Res., v.49, p.289-301, 2003.

DOUGHTERTY. C.T; COLLINS, M. Forage utilization. In: BARNES, R.F.; MILLER, D.A; NELSON,C.J. (Eds.). Forages: an introduction to grassland agriculture forages an introduction to glassland agriculture.6.ed. Ames: Iowa state University Press, 2003. p.391-414.

FARIA, V.P.; MATTOS, W.R.S. Nutrição de bovinos tendo em vista performances econômicas máximas. In: PEIXOTO, A.M., MOURA, J.C., FARIA, V.P. (Eds.). Nutrição de bovinos: conceitos básicos e aplicados. Piracicaba: FEALQ, 1995. p.199-222.

FERNANDES, J.J.R.; PIRES, A.V.; SANTOS, F.A.P. et al. Teores de caroço de algodão em dietas contendo silagem de milho para vacas em lactação. Acta Sci. Anim. Sci., v.24, p.1071-1077, 2002

GONÇALVES, L.C.; BORGES I.; FERREIRA, P.D.S. Alimentação de gado de leite. Belo Horizonte: FEPMVZ, 2009. 412p 
HESS, B.W.; MOSS, G.E.; RULE, D.C. A decade of developments in the area of fat supplementation research with beef cattle and sheep. J. Anim. Sci., v.86, p.188-204, 2008.

MENDOÇA, B.P.C.; LANA, R.P.; DETMANN, E. et al. Torta de crambe na terminação de bovinos de corte em confinamento. Arq. Bras. Med. Vet. Zootec., v.67, p.583-590, 2015.

MERTENS, D.R. Regulation of forage intake. In: FORAGE quality evaluation and utilization. Madison: American Society of Agronomy, Inc., 1994. p.450-493.

MIZUBUTI, I, Y.; RIBEIRO, E. L. A.; PEREIRA, E. S. et al. Cinética de fermentação ruminal in vitro de alguns co-produtos gerados na cadeia produtiva do biodiesel pela técnica de produção de gás. Semina Ciênc. Agr., v.32, p.2021-2028, 2011.

MIZUBUTI, I.Y., PINTO, A.P.P., RAMOS, B.M.O., PEREIRA, E.S. Métodos laboratoriais de avaliação de alimentos para animais. Londrina: EDUEL, 2009. 228 p.

PALMQUIST, D.L.; MATTOS, W.R.S. Metabolismo de lipídeos. In: BERCHIELLI, T.T.; PIRES, A.V.; OLIVEIRA, S.G. Nutrição de ruminantes. Jaboticabal: Funep, p.287-309, 2006.

PEREIRA, E.S.; MIZUBUTI, I.Y.; VILLARROEL, A.B.S.; OLIVEIRA, S.M. P. et al. Variáveis ruminais em novilhos alimentados com feno de Tifton 85 com diferentes tamanhos de partículas. Semina: Ciênc. Agr., v.30, p. 243-250. 2009.
SILVA, J.F.C; LEÃO, M. I. Fundamentos de nutrição dos ruminantes. Piracicaba: Livroceres, 1979. 380p.

SILVA, L.P.; NÖRNBERG, J.L. Prebióticos na nutrição de não ruminantes. Cienc. Rural, v.33, p.983-990, 2003.

SOUZA, A. D. V.; FÁVARO, S. P.; ÍTAVO, L. C.; ROSCOE, R. Caracterização química de sementes e tortas de pinhão manso, naboforrageiro e crambe. Pesquisa Agropecuária Brasileira, v.44, p.1328-1335, 2009

SNIFFEN, C.J; O'CONNOR, J.D.; VAN SOEST, P.J. FOX, D. G. et al. A net carbohydrate and protein system for evaluating cattle diets: II. Carbohydrate and protein availability. J. Anim. Sci., v.70, p.3562-3577, 1992.

THE NUTRITION of goats. Aberdeen: AFRC, 1998. (Report, 10).

URANO, F.S. Grãos de soja e bagaço de canade-açúcar in natura na alimentação de ovelhas da raça Santa Inês. 2009. 115f. Tese (Doutorado em Ciências) - Escola Superior de Agricultura Luiz de Queiroz, Piracicaba, SP.

VALADARES FILHO, S.C.; PINA, D.S. Fermentação ruminal. In: BERCHIELLI, T.T.; PIRES, A.V.; OLIVEIRA, S.G. (Eds.). Nutrição de ruminantes. Jaboticabal: FUNEP, 2006. p.151-182.

VAN SOEST, P.J. Nutritional ecology of the ruminant. 2.ed. Ithaca: Cornell University Press, p. 476,1994 